\title{
Prediction-Preserving Reducibility with Membership Queries on Formal Languages ${ }^{\star}$
}

\author{
Kouichi Hirata ${ }^{1}$ and Hiroshi Sakamoto ${ }^{2}$ \\ 1 Department of Artificial Intelligence, Kyushu Institute of Technology \\ Kawazu 680-4, Iizuka 820-8502, Japan \\ Tel: +81-948-29-7622, Fax: +81-948-29-7601 \\ hirata@ai.kyutech.ac.jp \\ 2 Department of Informatics, Kyushu University \\ Hakozaki 6-10-1, Fukuoka 812-8581, Japan \\ Tel: +81-92-642-2693, Fax: +81-92-642-2698 \\ hiroshi@i.kyushu-u.ac.jp
}

\begin{abstract}
This paper presents the prediction-preserving reducibility with membership queries (pwm-reducibility) on formal languages, in particular, simple CFGs and finite unions of regular pattern languages. For the former, we mainly show that DNF formulas are pwm-reducible to CFGs that is sequential or that contains at most one nonterminal. For the latter, we show that both bounded finite unions of regular pattern languages and unbounded finite unions of substring pattern languages are pwm-reducible to DFAs.
\end{abstract}

Keywords: prediction-preserving reduction with membership queries, prediction with membership queries, context-free grammars, pattern languages, grammatical inference, learning theory.

\section{Introduction}

The task of predicting the classification of a new example is frequently discussed from the viewpoints of both passive and active settings. In a passive setting, the examples are all chosen independently according to a fixed but unknown probability distribution, and the learner has no control over selection of examples [12, 18]. In an active setting, on the other hand, the learner is allowed to ask about particular examples, that is, the learner makes membership queries, before the new example to predict is given to the learner $[3,6]$.

Concerned with language learning, we can design a polynomial-time algorithm to predict deterministic finite automata (DFAs) in an active setting [3],

\footnotetext{
* This work is partially supported by Grant-in-Aid for Scientific Research (B) nos. 11558040, 11558084 and 13558036, and Grant-in-Aid for Young Scientists (A) no. 12780233. Also this paper will be published in Proc. of 13th International Symposium on Fundamentals of Computation Theory (FCT2001), Lecture Notes in Computer Science (C)Springer-Verlag.
} 
while predicting DFAs is as hard as computing certain apparently hard cryptographic predicates in a passive setting [12]. Furthermore, predicting nondeterministic finite automata (NFAs) and context-free grammars (CFGs) is also hard under the same cryptographic assumptions in an active setting [6]. Here, the cryptographic assumptions denote the intractability of inverting RSA encryption, recognizing quadratic residues and factoring Blum integers.

Pitt and Warmuth [18] have been formalized the model of a prediction and a reduction between two prediction problems that preserves polynomial-time predictability called the prediction-preserving reduction in a passive setting. Angluin and Kharitonov [6] have extended to the prediction and the reduction in an active setting. The reduction is called the prediction-preserving reduction with membership queries or a pwm-reduction for short.

The prediction is an weaker learning model than PAC-learning or query learning models [5, 6, 18]; If a class is polynomial-time learnable with equivalence (and membership) queries, then it is polynomial-time PAC-learnable (with membership queries), and if a class is polynomial-time PAC-learnable (with membership queries), then it is polynomial-time predictable (with membership queries).

Except the above general results, the detailed results of the pwm-reducibility on formal languages, for example, the restricted CFGs or another languages such as pattern languages, have few found elsewhere. Furthermore, many researchers have been interested in the pwm-reducibility on Boolean concepts but not on formal languages $[6,12,18]$. Hence, in this paper, we pay our attention to the pwm-reducibility on simple CFGs and finite unions of regular pattern languages.

For the former classes, we introduce several simple CFGs as follows: the linear grammars $\left(\mathcal{L}_{\text {linear }}\right)$, the right-linear grammars $\left(\mathcal{L}_{\text {right-linear }}\right)$, and the left-linear grammars $\left(\mathcal{L}_{\text {left-linear }}\right)$ as usual; the $k$-bounded $C F G s$ [4] $\left(\mathcal{L}_{k \text {-bounded-CFG }}\right)$ each of which right-hand side of productions contains at most $k$ nonterminals; the $s e$ quential CFGs $[7,24]\left(\mathcal{L}_{\mathrm{sqCFG}}\right)$ that the set of nonterminals has a partial order $\leq$ such that $T \rightarrow v U w$ iff $T \leq U$ for nonterminals $T$ and $U$; the properly sequential or loop-free $C F G s\left(\mathcal{L}_{\mathrm{psqCFG}}\right)$ that is sequential but disallowing to occur the same nonterminal in both left- and right-hand sides for each production; the $k-C F G s$ $\left(\mathcal{L}_{k \text {-CFG }}\right)$ that contains at most $k$ nonterminals; the parenthesis grammars [16] $\left(\mathcal{L}_{\text {paren }}\right)$ that each production is of the form $T \rightarrow[w]$.

On the other hand, a pattern [2] is a string consisting of constant symbols and variables, a regular pattern [25] is a pattern in which each variable occurs at most once, and a substring pattern [26] is a regular pattern of the form $x w y$ for a constant string $w$ and variables $x$ and $y$. The language of a pattern is the set of constant strings obtained by substituting nonempty constant strings for variables in the pattern. Then, for the latter classes, we deal with the bounded finite union of regular pattern languages by some constant $m\left(\mathcal{L}_{\cup_{m} R P}\right)$ and the unbounded finite union of regular or substring pattern languages $\left(\mathcal{L}_{\cup R P}\right.$ or $\left.\mathcal{L}_{\cup \text { subP }}\right)$.

We denote that $\mathcal{L}_{1}$ is pwm-reducible to $\mathcal{L}_{2}$ by $\mathcal{L}_{1} \unlhd_{\text {pwm }} \mathcal{L}_{2}$, and that $\mathcal{L}_{1} \unlhd_{\text {pwm }}$ $\mathcal{L}_{2}$ and $\mathcal{L}_{2} \unlhd_{\text {pwm }} \mathcal{L}_{1}$ by $\mathcal{L}_{1} \cong$ pwm $\mathcal{L}_{2}$. Then, this paper presents the results 
described as Fig. 1. Hence, we can conclude the following polynomial-time predictability with membership queries.

\begin{tabular}{|c|c|c|}
\hline $\mathcal{L}_{\mathrm{NFA}} \cong{ }_{\mathrm{pwm}}$ & $\mathcal{L}_{\text {right-linear }}, \mathcal{L}_{\text {left-linear }}$ & \\
\hline $\mathcal{L}_{\mathrm{NFA}} \unlhd_{\mathrm{pwm}}$ & $\mathcal{L}_{\text {linear }}, \mathcal{L}_{k \text {-bounded-CFG }}$ & \\
\hline $\mathcal{L}_{\mathrm{DNF}} \unlhd_{\mathrm{pwm}}$ & $\mathcal{L}_{\mathrm{sqCFG}}, \mathcal{L}_{\mathrm{psqCFG}}, \mathcal{L}_{k-\mathrm{CFG}}$ & \\
\hline $\mathcal{L}_{\cup \mathrm{DFA}} \unlhd_{\text {pwm }}$ & $\mathcal{L}_{\text {paren }}$ & \\
\hline & $\mathcal{L}_{\cup_{m} \mathrm{RP}}, \mathcal{L} \mathcal{L}_{\cup \text { subP }}$ & $\unlhd_{\text {pwm }} \mathcal{L}_{\text {DFA }}$ \\
\hline
\end{tabular}

Fig. 1. The pwm-reducibility

1. $\mathcal{L}_{\text {linear }}, \mathcal{L}_{\text {right-linear }}, \mathcal{L}_{\text {left-linear }}, \mathcal{L}_{k \text {-bounded-CFG }}(k \geq 1)$ and $\mathcal{L}_{\text {paren }}$ are not polynomial-time predictable with membership queries under the cryptographic assumptions.

2. If $\mathcal{L}_{\mathrm{sqCFG}}, \mathcal{L}_{\mathrm{psqCFG}}$ and $\mathcal{L}_{k \text {-CFG }}(k \geq 1)$ are polynomial-time predictable with membership queries, then so are DNF formulas.

3. $\mathcal{L}_{\cup_{m} \mathrm{RP}}(m \geq 0)$ and $\mathcal{L}_{\cup \text { subP }}$ are polynomial-time predictable with membership queries.

Concerned with the statement 2, this paper corrects and extends some results obtained by the paper [23]. Furthermore, concerned with the statement 3, we can show that $\mathcal{L}_{\mathrm{DNF}} \unlhd_{\text {pwm }} \mathcal{L}_{\cup R \mathrm{RP}}$, so if $\mathcal{L}_{\cup R P}$ is polynomial-time predictable with membership queries, then so are DNF formulas [22].

\section{Preliminaries}

Let $\Sigma$ and $N$ be two non-empty finite sets of symbols such that $\Sigma \cap N=\emptyset$. A production $A \rightarrow \alpha$ on $\Sigma$ and $N$ is an association from a nonterminal $A \in N$ to a string $\alpha \in(N \cup \Sigma)^{*}$. A context-free grammar (CFG, for short) is a 4-tuple $(N, \Sigma, P, S)$, where $S \in N$ is the distinguished start symbol and $P$ is a finite set of productions on $\Sigma$ and $N$. Symbols in $N$ are said to be nonterminals, while symbols in $\Sigma$ terminals. Then, we deal with the following subclasses of CFGs.

- A linear grammar is a CFG $G=(N, \Sigma, P, S)$ such that each production in $P$ is of the forms $T \rightarrow w U v$ or $T \rightarrow w$ for $T, U \in N$ and $w, v \in \Sigma^{*}$. In particular, a right-linear (resp., left-linear) grammar if it is a linear grammar such that each production is of the forms either $T \rightarrow w U$ (resp., $T \rightarrow U w$ ) or $T \rightarrow w$ for $T, U \in N$ and $w \in \Sigma^{*}$.

- A CFG $G=(N, \Sigma, P, S)$ is called $k$-bounded [4] if the right-hand side of each production in $P$ has at most $k$ nonterminals.

- A CFG $G=(N, \Sigma, P, S)$ is called sequential $[7,24]$ if the nonterminals in $N$ are labeled $S=T_{1}, \ldots, T_{n}$ such that, for each production $T_{i} \rightarrow w, w \in$ $\left(\Sigma \cup\left\{T_{j} \mid i \leq j \leq n\right\}\right)^{*}$. In particular, a sequential CFG satisfying that 
$w \in\left(\Sigma \cup\left\{T_{j} \mid i<j \leq n\right\}\right)^{*}$ for each production $T_{i} \rightarrow w$ is called properly sequential or loop-free.

- A CFG $G=(N, \Sigma, P, S)$ is called $a k-C F G$ if $|N| \leq k$.

- A parenthesis grammar [16] is a CFG $G=(N, \Sigma \cup\{[]\}, P, S$,$) such that each$ production in $P$ is of the form $T \rightarrow[w]$ for $T \in N$ and $w \in(N \cup \Sigma)^{*}$.

Let $G$ be a CFG $(N, \Sigma, S, P)$ and $\alpha$ and $\beta$ be strings in $(\Sigma \cup N)^{*}$. We denote $\alpha \Rightarrow_{G} \beta$ if there exist $\alpha_{1}, \alpha_{2} \in(\Sigma \cup N)^{*}$ such that $\alpha=\alpha_{1} X \alpha_{2}, \beta=\alpha_{1} \gamma \alpha_{2}$ and $X \rightarrow \gamma \in P$. We extend the relation $\Rightarrow_{G}$ to the reflexive and transitive closure $\Rightarrow_{G}^{*}$. For a nonterminal $A \in N$, the language $L_{G}(A)$ of $A$ is the set $\left\{w \in \Sigma^{*} \mid A \Rightarrow_{G}^{*} w\right\}$. The language $L(G)$ of $G$ just refers to $L_{G}(S)$.

Next, we introduce the notions of patterns [2]. Let $X$ be a countable set of variables such that $\Sigma \cap X=\emptyset$. A pattern is an element of $(\Sigma \cup X)^{+}$. A pattern $\pi$ is called regular [25] if each variables in $\pi$ occurs at most once. In particular, a regular pattern of the form $x w y$ is called a substring pattern [26] if $x, y \in X$ and $w \in \Sigma^{+}$.

A substitution is a homomorphism from patterns to patterns that maps each symbol $a \in \Sigma$ to itself. A substitution that maps some variables to an empty string $\varepsilon$ is called an $\varepsilon$-substitution. In this paper, we do not deal with $\varepsilon$-substitutions. By $\pi \theta$, we denote the image of a pattern by a substitution $\theta$. For a pattern $\pi$, the pattern language $L(\pi)$ is the set $\left\{w \in \Sigma^{+} \mid w=\right.$ $\pi \theta$ for some substitution $\theta$ \}.

\section{$3 \quad$ Prediction with Membership Queries}

In this section, we introduce definitions and theorems for prediction and predictionpreserving reduction with membership queries due to Angluin and Kharitonov [6].

Let $U$ denote $\Sigma^{*}$. If $w$ is a string, $|w|$ denotes its length. For each $n>0$, $U^{[n]}=\{w \in U|| w \mid \leq n\}$. A representation of concepts $\mathcal{L}$ is any subset of $U \times U$. We interpret an element $\langle u, w\rangle$ of $U \times U$ as consisting a concept representation $u$ and an example $w$. The example $w$ is a member of a concept $u$ if $\langle u, w\rangle \in \mathcal{L}$. Define the concept represented by $u$ as $\kappa_{\mathcal{L}}(u)=\{w \mid\langle u, w\rangle \in \mathcal{L}\}$. The set of concepts represented by $\mathcal{L}$ is $\left\{\kappa_{\mathcal{L}}(u) \mid u \in U\right\}$.

To represent CFGs, we define the class $\mathcal{L}_{\mathrm{CFG}}$ as the set of pairs $\langle u, w\rangle$ such that $u$ encodes a CFG $G$ and $w \in L(G)$. Also we define the classes $\mathcal{L}_{\text {linear }}$, $\mathcal{L}_{\text {right-linear }}, \mathcal{L}_{\text {left-linear }}, \mathcal{L}_{k \text {-bounded-CFG }}, \mathcal{L}_{\text {seqCFG }}, \mathcal{L}_{\text {psqCFG }}, \mathcal{L}_{k \text {-CFG }}$, and $\mathcal{L}_{\text {paren }}$, corresponding to linear grammars, right-linear grammars, left-linear grammars, $k$-bounded CFGs, sequential CFGs, properly sequential CFGs, $k$-CFGs, and parenthesis grammars, respectively, as similar.

To represent finite unions of regular pattern languages, we define the class $\mathcal{L}_{\cup_{m} \mathrm{RP}}$ as the set of pairs $\langle u, w\rangle$ such that $u$ encodes $m$ and a finite set $\pi_{1}, \cdots, \pi_{m}$ of $m$ regular patterns and $w$ is in the concept represented by $c$ iff $w \in L\left(\pi_{i}\right)$ for at least one $\pi_{i}$. Similarly, we define the class $\mathcal{L}_{\cup R P}\left(\right.$ resp., $\left.\mathcal{L}_{\cup \text { subP }}\right)$ as the set of pairs $\langle u, w\rangle$ such that $u$ encodes a finite set $\pi_{1}, \cdots, \pi_{r}$ of regular (resp., substring) patterns and $w$ is in the concept represented by $c$ iff $w \in L\left(\pi_{i}\right)$ for at 
least one $\pi_{i}$. Note that $\mathcal{L}_{\cup_{m} \mathrm{RP}}$ denotes the bounded finite unions, whereas $\mathcal{L} \cup \mathrm{RP}$ and $\mathcal{L}_{\cup \text { subP }}$ denote the unbounded finite unions.

Additionally, we introduce the following classes. The class $\mathcal{L}_{\mathrm{DFA}}\left(\right.$ resp., $\left.\mathcal{L}_{\mathrm{NFA}}\right)$ denotes the set of pairs $\langle u, w\rangle$ such that $u$ encodes a DFA (resp., NFA) $M$ and $M$ accepts $w$. The class $\mathcal{L} \cup$ DFA of finite union of DFAs denotes the set of pairs $\langle u, w\rangle$ such that $u$ encodes a finite set $M_{1}, \cdots, M_{r}$ of DFAs and $w$ is in the concept represented by $c$ iff at least one $M_{i}$ accepts $w$. The class $\mathcal{L}_{\text {DNF }}$ denotes the set of pairs $\langle u, w\rangle$ such that $u$ encodes a positive integer $n$ and a DNF formula $d$ over $n$ Boolean variables $x_{1}, \cdots, x_{n}$ such that $|w|=n\left(w=w_{1} \cdots w_{n}\right)$ and the assignment $x_{i}=w_{i}(1 \leq i \leq n)$ satisfies $d$.

Angluin and Kharitonov [6] have generalized the definitions of Pitt and Warmuth of prediction algorithm [18] to allow membership queries as follows.

Definition 1 (Angluin \& Kharitonov [6]). A prediction with membership queries algorithm, or pwm-algorithm, is a possibly randomized algorithm $A$ that takes as input $n$ (a bound on the size of examples), $s$ (a bound on the size of the target concept representations), and $\epsilon$ (an accuracy bound). It may make three different kinds of oracle calls, the responses to which are determined by the unknown target concept $c_{*}$ and the unknown distribution $D$ on $U^{[n]}$.

1. A membership query $[3,6]$ takes a string $w \in U$ as input and returns 1 if $w \in c_{*}$; and 0 otherwise.

2. A request for a random classified example takes as no input and returns a pair $\langle w, b\rangle$, where $w$ is a string chosen independently according to $D$ and $b=1$ if $x \in c_{*}$ and $b=0$ otherwise.

3. A request for an element to predict takes no input and returns a string $w$ chosen independently according to $D$.

$A$ may make any number of membership queries or requests for random classified examples, whereas $A$ must eventually make one and only one request for an element to predict and then eventually halt with an output 0 or 1 without making any further oracle calls. The output is interpreted as $A$ 's guess of how the target concept classifies the element returned by the request for an element to predict. A runs in polynomial time if its running time (counting one step per oracle call) is bounded by a polynomial in $n, s$ and $1 / \epsilon$.

Definition 2 (Angluin \& Kharitonov [6]). Let $\mathcal{L}$ be a representation of concepts and $c_{*}$ be the unknown target concept in $\mathcal{L}$. We say that $A$ successfully predicts $\mathcal{L}$ if, for each positive integer $n$ and $s$, for each positive rational $\epsilon$, for each concept representation $u \in U^{[n]}$, for each probability distribution $D$ on $U^{[n]}$, when $A$ is run with input $n, s$ and $\epsilon$, and oracles determined by $c_{*}=\kappa_{\mathcal{L}}(u)$ and $D, A$ asks membership queries that are in $U$ and the probability in at most $\epsilon$ that the output of $A$ is not equal to the correct classification of $w$ by $\kappa_{\mathcal{L}}(u)$, where $w$ is the string returned by the (unique) request for an element of predict.

Definition 3 (Angluin \& Kharitonov [6]). A representation $\mathcal{L}$ of concepts is polynomial-time predictable with membership queries if there exists a pwmalgorithm $A$ that runs in polynomial time and successfully predicts $\mathcal{L}$. 
It is well known the following statements:

1. $\mathcal{L}_{\mathrm{DFA}}$ is polynomial-time predictable with membership queries [3].

2. $\mathcal{L}_{\cup \mathrm{DFA}}, \mathcal{L}_{\mathrm{NFA}}$ and $\mathcal{L}_{\mathrm{CFG}}$ are not polynomial-time predictable with membership queries under the cryptographic assumptions [6].

3. $\mathcal{L}_{\mathrm{DNF}}$ is either polynomial-time predictable or not polynomial-time predictable with membership queries, if there exist one-way functions that cannot be inverted by polynomial-sized circuits [6].

Definition 4 (Angluin \& Kharitonov [6]). Let $\mathcal{L}_{i}$ be a representation of concepts over domain $U_{i}(i=1,2)$. We say that predicting $\mathcal{L}_{1}$ reduces to predicting $\mathcal{L}_{2}$ with membership queries (pwm-reduces, for short), denoted by $L_{1} \unlhd_{\text {pwm }} \mathcal{L}_{2}$, if there exist an instance mapping $f: \mathbf{N} \times \mathbf{N} \times U_{1} \rightarrow U_{2}$, a concept mapping $g: \mathbf{N} \times \mathbf{N} \times \mathcal{L}_{1} \rightarrow \mathcal{L}_{2}$, and a query mapping $h: \mathbf{N} \times \mathbf{N} \times U_{2} \rightarrow U_{1} \cup\{\top, \perp\}$ satisfying the following conditions.

1. For each $x \in U_{1}^{[n]}$ and $u \in \mathcal{L}_{1}^{[s]}, x \in \kappa_{\mathcal{L}_{1}}(u)$ iff $f(n, s, x) \in \kappa_{\mathcal{L}_{2}}(g(n, s, u))$.

2. $f$ is computable in time bounded by a polynomial in $n, s$ and $|x|$.

3. The size of $g(n, s, u)$ is bounded by a polynomial in $n, s$ and $|u|$.

4. For each $x^{\prime} \in U_{2}$ and $u \in \mathcal{L}_{1}^{[s]}$, if $h\left(n, s, x^{\prime}\right)=\top$ then $x^{\prime} \in \kappa_{\mathcal{L}_{2}}(g(n, s, u))$; if $h\left(n, s, x^{\prime}\right)=\perp$ then $x^{\prime} \notin \kappa_{\mathcal{L}_{2}}(g(n, s, u))$; if $h\left(n, s, x^{\prime}\right)=x \in U_{1}$, then it holds that $x^{\prime} \in \kappa_{\mathcal{L}_{2}}(g(n, s, u))$ iff $x \in \kappa_{\mathcal{L}_{1}}(u)$.

5. $h$ is computable in time bounded by a polynomial in $n, s$ and $\left|x^{\prime}\right|$.

Furthermore, we denote that $\mathcal{L}_{1} \unlhd_{\text {pwm }} \mathcal{L}_{2}$ and $\mathcal{L}_{2} \unlhd_{\text {pwm }} \mathcal{L}_{1}$ by $\mathcal{L}_{1} \cong$ pwm $\mathcal{L}_{2}$.

The following theorem is useful for showing the predictability or the hardness of predictability of the representations of concepts.

Theorem 1 (Angluin \& Kharitonov [6]). Let $\mathcal{L}_{1}$ and $\mathcal{L}_{2}$ be representations of concepts and suppose that $\mathcal{L}_{1} \unlhd_{\text {pwm }} \mathcal{L}_{2}$.

1. If $\mathcal{L}_{2}$ is polynomial-time predictable with membership queries, then so is $\mathcal{L}_{1}$.

2. If $\mathcal{L}_{1}$ is not polynomial-time predictable with membership queries, then neither is $\mathcal{L}_{2}$.

\section{Prediction-Preserving Reducibility with Membership Queries}

In this section, we fix $f, g$ and $h$ to an instance mapping, a concept mapping, and a query mapping. Furthermore, the parameters $n$ and $s$ denote the bounds of examples and representations, respectively. For simplicity, we assume that the length of examples for Boolean concepts is always fixed to the upper bound $n$. 


\subsection{Simple CFGs}

First of all, by using the transformation from a DFA to a right-linear grammar (cf. $[8,9]$ ), it holds that $\mathcal{L}_{\mathrm{DFA}} \unlhd_{\text {pwm }} \mathcal{L}_{\text {right-linear }}$, because the size of the right-linear grammar is bounded by a polynomial in the size of a DFA. Note that the converse direction $\mathcal{L}_{\text {right-linear }} \unlhd_{\text {pwm }} \mathcal{L}_{\text {DFA }}$ does not follows from the transformation from a right-linear grammar to a DFA, because the size of the DFA is not bounded by a polynomial in the size of a right-linear grammar in general.

Furthermore, we point out that $\mathcal{L}_{\mathrm{DNF}} \unlhd_{\text {pwm }} \mathcal{L}_{\cup \mathrm{DFA}}$, while $\mathcal{L}_{\mathrm{DNF}} \unlhd \mathcal{L}_{\mathrm{DFA}}[18]$. Here, the $\unlhd$ means the prediction-preserving reduction without membership queries introduced by Pitt and Warmuth [18], that is, there exist $f$ and $g$ satisfying the requirement from 1 to 3 in Definition 4 . Note that we cannot apply the same proof of $\mathcal{L}_{\mathrm{DNF}} \unlhd \mathcal{L}_{\mathrm{DFA}}$ to proving $\mathcal{L}_{\mathrm{DNF}} \unlhd_{\text {pwm }} \mathcal{L}_{\mathrm{DFA}}$; In this case, we cannot construct $h$ correctly.

On the other hand, by regarding the equivalent transformation between an NFA and a right-linear grammar $[8,9]$ as a concept mapping $g$, we observe that $\mathcal{L}_{\mathrm{NFA}} \cong_{\text {pwm }} \mathcal{L}_{\text {right-linear. }}$. Furthermore, for a CFG $G=(N, \Sigma, P, S)$, let $G^{R}$ be a CFG $\left(N, \Sigma, P^{\prime}, S\right)$ such that $T \rightarrow w^{R} \in P^{\prime}$ for each $T \rightarrow w \in P$. Here, $R$ denotes the reversal of a word. Then, for a right-linear (resp., left-linear) grammar $G$, construct the following $f, g$ and $h$ :

$$
\begin{aligned}
f(n, s, e) & =e^{R}, \\
g(n, s, G) & =G^{R}, \\
h\left(n, s, e^{\prime}\right) & =e^{\prime R} .
\end{aligned}
$$

It is obvious that $\mathcal{L}_{\text {right-linear }} \unlhd_{\text {pwm }} \mathcal{L}_{\text {left-linear }}$ (resp., $\mathcal{L}_{\text {left-linear }} \unlhd_{\text {pwm }} \mathcal{L}_{\text {right-linear }}$ ), so it holds that $\mathcal{L}_{\text {right-linear }} \cong_{\text {pwm }} \mathcal{L}_{\text {left-linear }}$. Similarly, we also observe that $\mathcal{L}_{\text {NFA }} \unlhd_{\text {pwm }} \mathcal{L}_{\text {linear }}$ and $\mathcal{L}_{\text {NFA }} \unlhd_{\text {pwm }} \mathcal{L}_{k \text {-bounded-CFG }}$ for each $k \geq 1$. Summary:

Theorem 2. $\mathcal{L}_{\mathrm{NFA}} \cong_{\text {pwm }} \mathcal{L}$ for $\mathcal{L} \in\left\{\mathcal{L}_{\text {right-linear }}, \mathcal{L}_{\text {left-linear }}\right\}$. Also, $\mathcal{L}_{\mathrm{NFA}} \unlhd_{\text {pwm }}$ $\mathcal{L}_{\text {linear }}$ and $\mathcal{L}_{\mathrm{NFA}} \unlhd_{\text {pwm }} \mathcal{L}_{k \text {-bounded-CFG }}$ for each $k \geq 1$.

Theorem 3. $\mathcal{L}_{\mathrm{DNF}} \unlhd_{\mathrm{pwm}} \mathcal{L}$ for $\mathcal{L} \in\left\{\mathcal{L}_{\mathrm{psqCFG}}, \mathcal{L}_{\mathrm{sqCFG}}\right\}$.

Proof. Let $d$ be a DNF formula $t_{1} \vee \cdots \vee t_{m}$ over $n$ Boolean variables $x_{1}, \ldots, x_{n}$. First, we define $w_{i}^{j}(1 \leq i \leq n, 1 \leq j \leq m)$ as follows:

$$
w_{i}^{j}= \begin{cases}1 & \text { if } t_{j} \text { contains } x_{i}, \\ 0 & \text { if } t_{j} \text { contains } \overline{x_{i}}, \\ T & \text { otherwise }\end{cases}
$$

Then, construct $f, g$ and $h$ as follows:

$$
\begin{aligned}
& f(n, s, e)=e, \\
& g(n, s, d)=\left(\{S, T\},\{0,1\}, S,\left\{S \rightarrow w_{1}^{1} \cdots w_{n}^{1}|\cdots| w_{1}^{m} \cdots w_{n}^{m}, T \rightarrow 0 \mid 1\right\}\right), \\
& h\left(n, s, e^{\prime}\right)=e^{\prime} .
\end{aligned}
$$

Note that $g(n, s, d)$ is a properly sequential CFG. It is obvious that the above $f, g$ and $h$ satisfy the conditions of Definition 4 . 
Theorem 4. For each $k \geq 1, \mathcal{L}_{\mathrm{DNF}} \unlhd_{\mathrm{pwm}} \mathcal{L}_{k-\mathrm{CFG}}$.

Proof. Theorem 3 implies that $\mathcal{L}_{\mathrm{DNF}} \unlhd_{\mathrm{pwm}} \mathcal{L}_{k \text {-CFG }}$ for each $k \geq 2$, so it is sufficient to show that $\mathcal{L}_{\mathrm{DNF}} \unlhd_{\text {pwm }} \mathcal{L}_{1-\mathrm{CFG}}$. Let $d=t_{1} \vee \cdots \vee t_{m}$ be a DNF formula over $n$ Boolean variables $x_{1}, \ldots, x_{n}$. First, define $w_{i}^{j}(1 \leq i \leq n, 1 \leq j \leq m)$ as follows:

$$
w_{i}^{j}= \begin{cases}1 & \text { if } t_{j} \text { contains } x_{i}, \\ 0 & \text { if } t_{j} \text { contains } \overline{x_{i}}, \\ S & \text { otherwise }\end{cases}
$$

Then, construct $f, g$ and $h$ as follows:

$$
\begin{aligned}
f(n, s, e)=e, & \\
g(n, s, d)= & \{\{S\},\{0,1\}, S, \\
& \left.\{S \rightarrow 0|1| w_{1}^{1} \cdots w_{n}^{1}|\cdots| w_{1}^{m} \cdots w_{n}^{m}|\underbrace{S \cdots S}_{n+1}| \cdots \mid \underbrace{S \cdots S}_{2 n}\}\right), \\
h\left(n, s, e^{\prime}\right)= & \begin{cases}e^{\prime} & \text { if }\left|e^{\prime}\right|=n, \\
\perp & \text { if } 1<\left|e^{\prime}\right|<n, \\
\top & \text { if }\left|e^{\prime}\right|=1 \text { or }\left|e^{\prime}\right|>n .\end{cases}
\end{aligned}
$$

For each $e \in\{0,1\}^{n}$, it holds that $e$ satisfies $d$ iff $S \Rightarrow_{g(n, s, d)}^{*} f(n, s, e)$. Furthermore, for each $e^{\prime} \in\{0,1\}^{*}$, if $h\left(n, s, e^{\prime}\right)=\perp$, then $S \nRightarrow_{g(n, s, d)}^{*} e^{\prime}$, because $g(n, s, d)$ generates no strings of length more than 1 and less than $n$; If $h\left(n, s, e^{\prime}\right)=e^{\prime}$, then it holds that $S \Rightarrow_{g(n, s, d)}^{*} e^{\prime}$ iff $h\left(n, s, e^{\prime}\right)$ satisfies $d$.

Finally, consider the case that $h\left(n, s, e^{\prime}\right)=T$. It is sufficient to show that, for each $k \geq 1$, it holds that $S \Rightarrow_{g(n, s, d)}^{*} \underbrace{S \cdots S}_{k n+m}$ for each $m(1 \leq m \leq n)$. If $k=1$, then, by the definition, it holds that $S \Rightarrow_{g(n, s, d)}^{*} \underbrace{S \cdots S}_{n+m}$ for each $m(1 \leq m \leq n)$. Suppose that, for some $k \geq 1, S \Rightarrow_{g(n, s, d)}^{*} \underbrace{S \cdots S}_{k n+m}$ for each $m(1 \leq m \leq n)$. Then, it holds that $S \Rightarrow_{g(n, s, d)}^{*} \underbrace{S \cdots S}_{k n+(m-1)} S \Rightarrow_{g(n, s, d)} \underbrace{S \cdots S}_{k n+(m-1)} \underbrace{S \cdots S}_{n+1}=\underbrace{S \cdots S}_{(k+1) n+m}$ for each $m(1 \leq m \leq n)$. Hence, $g(n, s, d)$ generates all strings of length more than $n$, so if $h\left(n, s, e^{\prime}\right)=\top$, then $S \Rightarrow_{g(n, s, d)}^{*} e^{\prime}$.

Theorem 5. $\mathcal{L}_{\cup D F A} \unlhd_{\text {pwm }} \mathcal{L}_{\text {paren }}$.

Proof. Let $M_{1}, \ldots, M_{r}$ be DFAs with the same alphabet $\Sigma([,] \notin \Sigma)$ and with mutually distinct states. For each $M_{i}=\left(Q_{i}, \Sigma, \delta_{i}, q_{0}^{i}, F_{i}\right)(1 \leq i \leq r)$, construct a parenthesis grammar $G_{i}\left(n, s, M_{i}\right)=\left(Q_{i}, \Sigma \cup\{[]\},, P_{i}, q_{0}^{i}\right)$ such that $q \rightarrow\left[a \delta_{i}(q, a)\right] \in P_{i}$ for each $q \in Q_{i}$ and $a \in \Sigma ; q \rightarrow[\varepsilon]$ for each $q \in F_{i}$, where $\varepsilon$ is an empty string. By using $G_{i}\left(n, s, M_{i}\right)$, let $P_{M_{1}, \ldots, M_{r}}$ be the following set of productions for $S \notin\left(\cup_{1 \leq i \leq r} Q_{i}\right) \cup \Sigma \cup\{[]$,$\} :$

$$
P_{M_{1}, \ldots, M_{r}}=\left\{S \rightarrow\left[q_{0}^{1}\right]|\cdots|\left[q_{0}^{r}\right]\right\} \cup\left(\cup_{1 \leq i \leq r} P_{i}\right) .
$$

Then, construct $f, g$ and $h$ as follows: 


$$
\begin{aligned}
f\left(n, s, e_{1} e_{2} \cdots e_{l}\right) & =\left[\left[e_{1}\left[e_{2}\left[\cdots\left[e_{l}[\varepsilon]\right] \cdots\right]\right]\right]\right] \text { for } e_{i} \in \Sigma, \\
g\left(n, s,\left\{M_{1}, \ldots, M_{r}\right\}\right) & =\left(\{S\} \cup\left(\cup_{1 \leq i \leq r} Q_{i}\right), \Sigma \cup\{[,]\}, S, P_{M_{1}, \ldots, M_{r}}\right), \\
h\left(n, s, e^{\prime}\right) & =\left\{\begin{array}{cl}
e_{1} \cdots e_{l} \text { if } e^{\prime}=\left[\left[e_{1}\left[e_{2}\left[\cdots\left[e_{l}[\varepsilon]\right] \cdots\right]\right]\right]\right] \text { and } e_{i} \in \Sigma, \\
\perp & \text { otherwise. }
\end{array}\right.
\end{aligned}
$$

Note that $L\left(g\left(n, s,\left\{M_{1}, \ldots, M_{r}\right\}\right)\right) \subseteq\left\{\left[\left[e_{1}\left[e_{2}\left[\cdots\left[e_{m}[\varepsilon]\right] \cdots\right]\right]\right]\right] \mid m \geq 1, e_{i} \in\right.$ $\Sigma\}$, so if $h\left(n, s, e^{\prime}\right)=\perp$, then $S \nRightarrow_{g\left(n, s,\left\{M_{1}, \ldots, M_{r}\right\}\right)}^{*} e^{\prime}$. Also it is obvious that $S \Rightarrow_{g\left(n, s,\left\{M_{1}, \ldots, M_{r}\right\}\right)}^{*}\left[\left[e_{1}\left[e_{2}\left[\cdots\left[e_{l}[\varepsilon]\right] \cdots\right]\right]\right]\right]$ iff $e_{1} e_{2} \cdots e_{l} \in L\left(M_{i}\right)$ for some $i(1 \leq$ $i \leq r)$. Hence, it holds that $\mathcal{L}_{\mathrm{UDFA}} \unlhd_{\text {pwm }} \mathcal{L}_{\text {paren }}$.

Sakakibara [19] has shown that $\mathcal{L}_{\text {paren }}$ is polynomial-time learnable with membership and equivalence queries if the structural information is available. Furthermore, Sakamoto [21] has shown that $\mathcal{L}_{\text {paren }}$ is polynomial-time learnable with membership queries and characteristic examples. The above theorem claims that the structural information or the characteristic examples are essential for efficient learning of $\mathcal{L}_{\text {paren }}$.

\subsection{Finite unions of regular pattern languages}

Since each regular pattern language is regular [25], we can construct a DFA $M_{\pi}$ such that $L\left(M_{\pi}\right)=L(\pi)$ for each regular pattern $\pi$ as follows: Suppose that $\pi$ is a regular pattern of the form $\pi=x_{0} \alpha_{1} x_{1} \alpha_{2} \cdots x_{n-1} \alpha_{n} x_{n}$, where $x_{i} \in X$ and $\alpha_{i}=a_{1}^{i} a_{2}^{i} \cdots a_{m_{i}}^{i} \in \Sigma^{+}$. Then, the corresponding DFA for $\pi$ is the DFA $M_{\pi}=\left(\Sigma, Q, \delta, q_{0}, F\right)$ such that:

1. $Q=\left\{q_{0}, p_{1}^{1}, \ldots, p_{m_{1}}^{1}, q_{1}, p_{1}^{2}, \ldots, p_{m_{2}}^{2}, q_{2}, \ldots, q_{n-1}, p_{1}^{n}, \ldots, p_{m_{n}}^{n}, q_{n}\right\}$ and $F=$ $\left\{q_{n}\right\}$

2. $\delta\left(q_{i}, a\right)=p_{1}^{i+1}$ and $\delta\left(q_{n}, a\right)=q_{n}$ for each $a \in \Sigma$ and $0 \leq i \leq n-1$,

3. $\delta\left(p_{j}^{i}, a_{j}^{i}\right)=p_{j+1}^{i}$ and $\delta\left(p_{m_{i}}^{i}, a_{m_{i}}^{i}\right)=q_{i}$ for each $1 \leq i \leq n$ and $1 \leq j \leq m_{i}-1$,

4. $\delta\left(p_{j}^{i}, a\right)=p_{1}^{i}$ for each $a \in \Sigma$ such that $a \neq a_{j}^{i}$.

It is obvious that $\left|M_{\pi}\right|$ is bounded by a polynomial in $|\pi|$.

By using the corresponding DFAs, we can easily shown that $\mathcal{L}_{\mathrm{RP}} \unlhd_{\text {pwm }} \mathcal{L}_{\mathrm{DFA}}$ by constructing the following $f, g$ and $h$ for each regular pattern $\pi$ :

$$
\begin{gathered}
f(n, s, e)=e, \\
g(n, s, \pi)=M_{\pi}, \\
h\left(n, s, e^{\prime}\right)=e^{\prime} .
\end{gathered}
$$

Then, $\mathcal{L}_{\mathrm{RP}}$ is polynomial-time predictable with membership queries, which is implied by the result of Matsumoto and Shinohara [15] that $\mathcal{L}_{\mathrm{RP}}$ is polynomial-time learnable with equivalence and membership queries. Furthermore, the following theorem holds:

Theorem 6. For each $m \geq 0, \mathcal{L}_{\cup_{m} \mathrm{RP}} \unlhd_{\text {pwm }} \mathcal{L}_{\mathrm{DFA}}$.

Proof. Let $\pi_{1}, \ldots, \pi_{m}$ be $m$ regular patterns. Also let $M_{\pi_{i}}=\left(Q_{i}, \Sigma, \delta_{i}, q_{0}^{i}, F_{i}\right)$ be the corresponding DFA of $\pi_{i}$. First, construct a DFA $M_{\pi_{1}, \ldots, \pi_{m}}=\left(Q_{1} \times \cdots \times\right.$ $\left.Q_{m}, \Sigma, \delta,\left(q_{0}^{1}, \ldots, q_{0}^{m}\right), F_{1} \times \cdots \times F_{m}\right)$ such that $\delta\left(\left(q_{1}, \ldots, q_{m}\right), a\right)=\left(p_{1}, \ldots, p_{m}\right)$ iff $\delta_{i}\left(q_{i}, a\right)=p_{i}$ for each $i(1 \leq i \leq m)$. Then, construct $f, g$ and $h$ as follows: 


$$
\begin{aligned}
f(n, s, e) & =e \\
g\left(n, s,\left\{\pi_{1}, \ldots, \pi_{m}\right\}\right) & =M_{\pi_{1}, \ldots, \pi_{m}}, \\
h\left(n, s, e^{\prime}\right) & =e^{\prime} .
\end{aligned}
$$

Recall that the size of $g\left(n, s,\left\{\pi_{1}, \ldots, \pi_{m}\right\}\right)$ is bounded by a polynomial in $s$, that is, $O\left(s^{m}\right)$. It is obvious that $L\left(\pi_{1}\right) \cup \cdots \cup L\left(\pi_{m}\right)=L\left(M_{\pi_{1}, \ldots, \pi_{m}}\right)$, which implies that $\mathcal{L}_{\cup_{m} R P} \unlhd_{\text {pwm }} \mathcal{L}_{\text {DFA }}$.

For the unbounded finite unions of regular pattern languages, the following theorem holds from our previous result.

Theorem 7 (Sakamoto et al. [22]). $\mathcal{L}_{\mathrm{DNF}} \unlhd_{\text {pwm }} \mathcal{L}_{\cup \mathrm{RP}}$.

The idea of the proof of Theorem 7 in [22] is similar as one of Theorem 4: First, for each term $t_{j}(1 \leq j \leq m)$ in a DNF formula $d=t_{1} \vee \cdots \vee t_{m}$ over $x_{1}, \ldots, x_{n}$, construct a regular pattern $\pi_{j}=\pi_{1}^{j} \cdots \pi_{n}^{j}$ as follows:

$$
\pi_{i}^{j}= \begin{cases}1 & \text { if } t_{j} \text { contains } x_{i}, \\ 0 & \text { if } t_{j} \text { contains } \overline{x_{i}}, \\ x_{i}^{j} & \text { otherwise }\end{cases}
$$

Furthermore, let $\pi$ be a regular pattern $x_{1} \cdots x_{n} x_{n+1}$. Then, we can construct $f$ and $h$ as same as Theorem 4 and $g$ as $g(n, s, d)=\left\{\pi_{1}, \ldots, \pi_{m}, \pi\right\}$. Note that $L(\pi)=\Sigma^{*}-\Sigma^{[n]}$, because of disallowing $\varepsilon$-substitutions.

For the unbounded finite unions of substring pattern languages, we obtain the following theorem.

Theorem 8. $\mathcal{L}_{\cup \text { subP }} \unlhd_{\text {pwm }} \mathcal{L}_{\text {DFA }}$.

Proof. Let $\pi_{1}, \ldots, \pi_{r}$ be substring patterns such that $\pi_{i}=x_{i} w_{i} y_{i}$. For a set $\left\{w_{1}, \ldots, w_{r}\right\}$ of constant strings, consider the following modification of a pattern matching machine (pmm) $M_{w_{1}, \ldots, w_{r}}$ [1]. The goto function is defined as same as a pmm. For the state $j$ transitioned from the right-most constant of $w_{i}$ by the goto function, the failure function maps $j$ to $j$ itself; otherwise, the failure function is defined as same as a pmm. The output function is not necessary.

Since this modified pmm is also a DFA, construct $f, g$ and $h$ as follows:

$$
\begin{aligned}
f(n, s, e) & =e, \\
g\left(n, s,\left\{\pi_{1}, \ldots, \pi_{r}\right\}\right) & =M_{w_{1}, \ldots, w_{r}}, \\
h\left(n, s, e^{\prime}\right) & =e^{\prime} .
\end{aligned}
$$

Note that the size of $g\left(n, s,\left\{\pi_{1}, \ldots, \pi_{r}\right\}\right)$ is $O\left(\left|w_{1}\right|+\cdots+\left|w_{r}\right|\right)$ [1]. Furthermore, it is obvious that $L\left(g\left(n, s,\left\{\pi_{1}, \ldots, \pi_{r}\right\}\right)\right)=L\left(\pi_{1}\right) \cup \cdots \cup L\left(\pi_{r}\right)$, which implies that $\mathcal{L}_{\cup \text { subP }} \unlhd_{\text {pwm }} \mathcal{L}_{\text {DFA }}$.

Shinohara and Arimura [26] have discussed the inferability of $\mathcal{L}_{\cup_{m} R P}, \mathcal{L}_{\cup R P}$ and $\mathcal{L}_{\cup \text { subP }}$ in the framework of inductive inference. They have shown that $\mathcal{L}_{\cup_{m} \mathrm{RP}}$ and $\mathcal{L}_{\cup \text { subP }}$ are inferable from positive data in the limit, whereas $\mathcal{L}_{\cup R P}$ is not. In contrast, by Theorem 6,7 and $8, \mathcal{L}_{\cup_{m} \mathrm{RP}}$ and $\mathcal{L}_{\cup \text { subP }}$ are polynomial-time predictable with membership queries, whereas $\mathcal{L}_{\cup R P}$ is not if neither are DNF formulas. 


\section{Conclusion}

In this paper, we have presented the results of pwm-reducibility on formal languages described as Fig. 1 in Section 1.

The results in Section 4.1 tell us that the efficient predictability of CFGs may be necessary to assume some deterministic application for productions. Ishizaka [10] showed that the simple deterministic grammars are polynomialtime learnable with extended equivalence and membership queries. The extended equivalence query can check whether or not the hypothesis is generated by simple deterministic grammars. It remains open whether or not simple deterministic grammars are polynomial-time predictable with membership queries.

Angluin [4] showed that $\mathcal{L}_{k \text {-bounded-CFG is polynomial-time predictable with }}$ nonterminal membership queries, and Sakakibara [20] extended Angluin's result to extended simple formal systems. In our previous work, we extended the pwm-reduction to prediction-preserving reduction with nonterminal membership queries partially [22]. It is important to investigate the properties of it in more detail.

In Section 4.2, we only deal with the finite unions of regular pattern languages. Many researchers such as $[2,11,13-15,17,26]$ have developed the learnability/predictability of non-regular pattern languages. In particular, the learnability of the languages of $k$-variable patterns $[2,11]$ that contain at most $k$ variables, $k \mu$-patterns [15] each of which variable occurs at most $k$ times, and erasing patterns $[17,26]$ that allow $\varepsilon$-substitutions have been widely studied in the various learning frameworks. It is a future work to investigate the pwmreducibility of them or their finite unions.

\section{Acknowledgment}

The authors would like to thank Hiroki Arimura in Kyushu University for the idea of the proof of Theorem 8 and also valuable comments.

\section{References}

1. A. V. Aho, M. J. Corasick, Efficient string matching: An aid to bibliographic search, Comm. ACM 18(6) (1975), 333-340.

2. D. Angluin, Finding patterns common to a set of strings, J. Comput. System Sci. 21(1) (1980) 46-62.

3. D. Angluin, Learning regular sets from queries and counterexamples, Inform. Comput. 75(2) (1987) 87-106.

4. D. Angluin, Learning k-bounded context-free grammars, Technical Report YALEU/DCS/RR-557, Yale University, 1987.

5. D. Angluin, Queries and concept learning, Mach. Learn. 2(4) (1988) 319-342.

6. D. Angluin, M. Kharitonov, When won't membership queries help?, J. Comput. System Sci. 50(2) (1995) 336-355.

7. A. Ginsburg, The mathematical theory of context free languages (McGraw-Hill, 1966). 
8. M. A. Harrison, Introduction to formal language theory (Addison-Wesley, 1978).

9. J. E. Hopcroft, J. D. Ullman, Introduction to automata theory, languages and computation (Addison-Wesley, 1979).

10. H. Ishizaka, Polynomial time learnability of simple deterministic languages, Mach. Learn. 5(2) (1990) 151-164.

11. M. Kearns, L. Pitt, A polynomial-time algorithm for learning k-variable pattern languages from examples, in: Proc. 2nd Ann. Conf. on Computational Learning Theory (ACM, 1989) 57-71.

12. M. Kearns, L. Valiant, Cryptographic limitations on learning Boolean formulae and finite automata, J. ACM 41(1) (1994) 67-95.

13. A. Marron, Identification of pattern languages from examples and queries, Inform. Comput. 74(2) (1987) 91-112.

14. A. Marron, Learning pattern languages from a single initial example and from queries, in: Proc. 1st Ann. Workshop on Computational Learning Theory (ACM, 1988) 345-358.

15. S. Matsumoto, A. Shinohara, Learning pattern languages using queries, in: Proc. 3rd Euro. Conf. on Computational Learning Theory, LNAI 1208 (Springer, 1997) 185-197.

16. R. McNaughton, Parenthesis grammars, J. ACM 14(3) (1967) 490-500.

17. J. Nessel, S. Lange, Learning erasing pattern languages with queries, in: Proc. 11th Internat. Conf. on Algorithmic Learning Theory, LNAI 1968 (Springer, 2000) 86100.

18. L. Pitt, M. K. Warmuth, Prediction-preserving reduction, J. Comput. System Sci. 41(3) (1990) 430-467.

19. Y. Sakakibara, Learning context-free grammars from structural data in polynomial time, Theor. Comput. Sci. 76(2-3) (1990) 223-242.

20. Y. Sakakibara, On learning Smullyan's elementary formal systems: Towards an efficient learning method for context-sensitive languages, Adv. Soft. Sci. Tech. 2 (1990) 79-101.

21. H. Sakamoto, Language learning from membership queries and characteristic examples, in: Proc. 6th Internat. Workshop on Algorithmic Learning Theory, LNAI 997 (Springer, 1995) 55-65.

22. H. Sakamoto, K. Hirata, H. Arimura, Learning elementary formal systems with queries, Technical Report DOI-TR-179, Department of Informatics, Kyushu University, 2000. Also available at http://www.i.kyushu-u.ac.jp/doi-tr.html.

23. N. Sugimoto, T. Toyoshima, S. Shimozono, K. Hirata, Constructive learning of context-free languages with a subpansive tree, in: Proc. 5th Internat. Colloq. on Grammatical Inference, LNAI 1891 (Springer, 2000) 270-283.

24. E. Shamir, On sequential languages and two classes of regular events, Zeit. Phone., Sprach. und Kommun. 18 (1965) 61-69.

25. T. Shinohara, Polynomial time inference of extended regular pattern languages, in: Proc. RIMS Symposia on Software Science and Engineering, LNCS 147 (Springer, 1982) 115-127.

26. T. Shinohara, H. Arimura, Inductive inference of unbounded unions of pattern languages from positive data, Theor. Comput. Sci. 241 (2000) 191-209. 